Днепродзержинский государственный технический университет

\title{
O геометрии выпуклых поверхностей
}

Получено одно представлеиме ныпулых ограниченных поверхностей через

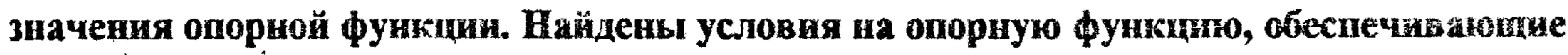
выпуклость поверхности, а также получены некоторые характеристики таких поверхностей.

В [2] приведено представление выпуклых кривых, которое оказалось достаточно эффективным и полезным при исследованик многих геометрических свойств плоских кривых. В частности, было доказано, что любая кривая $\Gamma(\varphi)$ представима в виде

$$
\Gamma(\theta, \varphi)=\left\{\begin{array}{l}
x(\theta, \varphi) \\
y(\theta, \varphi)
\end{array}=\left\{\begin{array}{l}
-\theta(\varphi) \sin \varphi-\theta^{\prime}(\varphi) \cos \varphi \\
\theta(\varphi) \cos \varphi-\theta^{\prime}(\varphi) \sin \varphi
\end{array}\right.\right.
$$

где функция $\theta(\varphi)$ такова, что $\theta(\varphi)+\theta^{\prime \prime}(\varphi)$ не меняет знак на периоде.

В данной работе предпринята попытка получить аналогичное представление выпуклых поверхностей.

Рассмотрим поверхность П $(\varphi, \psi)$, заданную равенствами

$$
\left\{\begin{array}{c}
x=x(\varphi, \psi)=\theta(\varphi, \psi) \sin \psi \cos \varphi+\theta_{\psi}^{\prime}(\varphi, \psi) \cos \psi \cos \varphi-\theta_{\varphi}^{\prime}(\varphi, \psi) \frac{\sin \varphi}{\sin \psi} \\
y=y(\varphi, \psi)=\theta(\varphi, \psi) \sin \psi \sin \varphi+\theta_{\psi}^{\prime}(\varphi, \psi) \cos \psi \sin \varphi+\theta_{\varphi}(\varphi, \psi) \frac{\cos \varphi}{\sin \psi} \\
z=z(\varphi, \psi)=\dot{\theta}(\varphi, \psi) \cos \psi-\theta_{\psi}^{\prime}(\varphi, \psi) \sin \psi
\end{array}\right.
$$

где $\theta(\varphi, \psi)$ строго положительная непрерывная вместе со своими производными функция, заданная на области $\varphi \in[0,2 \pi], \psi \in(0, \pi)$. Приведем некоторые свойства поверхностей вида (1).

Положим

$$
K_{\Pi}(\varphi, \psi)=\frac{\sin ^{2} \psi}{\left(\theta+\theta_{\psi \psi}^{\prime \prime}\right)\left(\theta \sin ^{2} \psi+\theta_{\psi}^{\prime} \cos \psi \sin \psi+\theta_{\varphi \varphi}^{\prime}\right)-\left(\theta_{\varphi}^{\prime} \operatorname{ctg} \psi-\theta_{\Phi \psi}^{\prime \prime}\right)^{2}},
$$

здесь и далее $\theta=\theta(\varphi, \psi)$. 
Предложение 1. Для люб́ых значений $\varphi \in[0,2 \pi], \psi \in(0, \pi)$ вектор $\overrightarrow{\mathbf{n}}^{0}\left(\mathrm{n}_{\mathrm{x}}^{0}, \mathrm{n}_{\mathrm{y}}^{0}, \mathrm{n}_{\mathrm{z}}^{0}\right)$, где

$$
\text { - } \mathrm{n}_{\mathrm{x}}^{0}=\cos \varphi \sin \psi, \quad \mathrm{n}_{\mathrm{y}}^{0}=\sin \varphi \sin \psi, \quad \mathrm{n}_{\mathrm{z}}^{0}=\cos \psi
$$

есть единичный вектор нормали к поверхности (1) в точке П $(\varphi, \psi)$, то есть для поверхности, представимой в виде (1), значения параметров $\varphi$ и $\psi$ есть углы Эйлера нормального вектора к поверхности.

Доказательство. Действительно, если $\frac{\partial(\mathrm{y}, \mathrm{z})}{\partial(\varphi, \psi)}, \frac{\partial(\mathrm{z}, \mathrm{x})}{\partial(\varphi, \psi)}$ и $\frac{\partial(\mathrm{x}, \mathrm{y})}{\partial(\varphi, \psi)}$. функциональные определители матрицы

$$
\left(\begin{array}{lll}
\frac{\partial x}{\partial \varphi} & \frac{\partial y}{\partial \varphi} & \frac{\partial z}{\partial \varphi} \\
\frac{\partial x}{\partial \psi} & \frac{\partial y}{\partial \psi} & \frac{\partial z}{\partial \psi}
\end{array}\right)
$$

то, как известно, вектор

$$
\frac{\partial(y, z)}{\partial(\varphi, \psi)} \vec{i}+\frac{\partial(z, x)}{\partial(\varphi, \psi)} \vec{j}+\frac{\partial(x, y)}{\partial(\varphi, \psi)} \vec{k}
$$

есть вектор нормали к поверхности $\mathrm{x}=\mathrm{x}(\varphi, \psi), \mathrm{y}=\mathrm{y}(\varphi, \psi)$ и $z=z(\varphi, \psi)$.

С другой стороны, имеют место равенства

$$
\begin{gathered}
\frac{\partial \mathrm{x}}{\partial \varphi}=\theta_{\varphi}^{\prime} \sin \psi \cos \varphi-\theta \sin \psi \sin \varphi+\theta_{\varphi \psi}^{\prime \prime} \cos \varphi \cos \psi- \\
-\theta_{\psi}^{\prime} \cos \psi \cos \varphi-\theta_{\varphi \psi}^{\prime \prime} \frac{\sin \varphi}{\sin \psi}-\theta_{\varphi}^{\prime} \frac{\cos \varphi}{\sin \psi}, \\
\frac{\partial \mathrm{x}}{\partial \psi}=\theta \cos \psi \cos \varphi+\theta_{\psi \psi}^{\prime \prime} \cos \varphi \cos \psi-\theta_{\varphi \psi}^{\prime \prime} \frac{\sin \varphi}{\sin \psi}+\theta_{\varphi}^{\prime} \frac{\sin \varphi \cos \psi}{\sin ^{2} \psi}, \\
\frac{\partial y}{\partial \varphi}=\theta_{\varphi}^{\prime} \sin \psi \sin \varphi+\theta \sin \psi \cos \varphi+\theta_{\varphi \psi}^{\prime} \sin \varphi \cos \psi+ \\
+\theta_{\psi}^{\prime} \cos \psi \cos \varphi+\theta_{\varphi \psi}^{\prime \prime} \frac{\cos \varphi}{\sin \psi}-\theta_{\varphi}^{\prime} \frac{\sin \varphi}{\sin \psi}, \\
\frac{\partial y}{\partial \psi}=\theta \cos \psi \sin \varphi+\theta_{\psi \psi}^{\prime \prime} \sin \varphi \cos \psi+\theta_{\varphi \psi}^{\prime \prime} \frac{\cos \varphi}{\sin \psi}-\theta_{\varphi}^{\prime} \frac{\cos \varphi \cos \psi}{\sin ^{2} \psi},
\end{gathered}
$$




$$
\frac{\partial z}{\partial \varphi}=\theta_{\varphi}^{\prime \prime} \cos \psi-\theta_{\varphi \psi}^{\prime \prime} \sin \psi, \quad \frac{\partial z}{\partial \psi}=-\theta \sin \psi-\theta_{\psi \psi}^{*} \sin \psi
$$

Отсюда легко получаем значения фунхциональных определителей

$$
\begin{aligned}
& \frac{\partial(\mathrm{y}, \mathrm{z})}{\partial(\varphi, \psi)}=-\cos \varphi\left(\left(\theta+\theta_{\psi \psi}^{\prime \prime}\right)\left(\theta \sin ^{2} \psi+\theta_{\psi}^{\prime} \cos \psi \sin \psi+\theta_{\psi \psi}^{\prime \prime}\right)-\left(\theta_{\varphi}^{\prime} \operatorname{ctg} \psi-\theta_{\varphi \psi}^{\prime}\right)^{2}\right) \\
& \frac{\partial(\mathrm{z}, \mathrm{x})}{\partial(\varphi, \psi)}=-\sin \varphi\left(\left(\theta+\theta_{\psi \psi}^{\prime \prime}\right)\left(\theta \sin ^{2} \psi+\theta_{\psi}^{\prime} \cos \psi \sin \psi+\theta_{\psi \psi}^{\prime \prime}\right)-\left(\theta_{\Psi}^{\prime} \operatorname{ctg} \psi-\theta_{\psi \psi}^{\prime \prime}\right)^{2}\right)
\end{aligned}
$$

и

$$
\frac{\partial(\mathrm{x}, \mathrm{y})}{\partial(\varphi, \psi)}=-\frac{\cos \psi}{\sin \psi}\left(\left(\theta+\theta_{\psi \psi}^{*}\right)\left(\theta \sin ^{2} \psi+\theta_{\psi}^{\prime} \cos \psi \sin \psi+\theta_{\psi \psi}^{*}\right)-\left(\theta_{\varphi}^{\prime} \operatorname{ctg} \psi-\theta_{\varphi \psi \psi}^{*}\right)^{2}\right)(
$$

Таким образом, вектор $\overrightarrow{\mathrm{N}}\left(\mathrm{N}_{\mathrm{x}}, \mathrm{N}_{\mathrm{y}}, \mathrm{N}_{\mathrm{z}}\right)$,

$$
N_{x}=\cos \varphi \sin \psi \frac{\sin \psi}{K_{\Pi}(\varphi, \psi)}, \quad N_{y}=\sin \varphi \sin \psi \frac{\sin \psi}{K_{\Pi}(\varphi, \psi)}, \quad N_{z}=\cos \psi \frac{\cdot \sin \psi}{K_{\Pi}(\varphi, \psi)}
$$

есть нормальный вектор поверхности (1). Ясно, что единичный вектор нормали есть вектор (3).

Предложение 2. Для любых значений $\varphi \in[0,2 \pi]$ и $\psi \in(0, \pi)$ Гауссова кривизна поверхности П( $\varphi, \psi)$, определенной равенствами (1), равна $\mathrm{K}_{\Pi}(\varphi, \psi)$, а значение средней кривизны поверхности $\Pi(\varphi, \psi)$ имеет вид

$$
\mathrm{H}_{\Pi}(\varphi, \psi)=-\frac{1}{2} \frac{\left(2 \theta+\theta_{\psi \psi}^{*}\right) \sin ^{2} \psi+\theta_{\psi}^{\prime} \cos \psi \sin \psi+\theta_{\Phi \varphi}^{*}}{\left(\theta+\theta_{\psi \psi}^{*}\right)\left(\theta \sin ^{2} \psi+\theta_{\psi}^{\prime} \cos \psi \sin \psi+\theta_{\varphi \varphi}^{*}\right)-\left(\theta_{\Phi}^{\prime} \operatorname{ctg} \psi-\theta_{\phi \psi}^{*}\right)^{2}}
$$

Доказательство . Как известно (см., напр. , [1]), значепие Гауссовой кривизны поверхности определяется равенством

$$
K_{\Pi}(\varphi, \psi)=\frac{b_{11} b_{22}-b_{12}^{2}}{g_{11} g_{22}-g_{12}^{2}}
$$

а значение средней кривизны равно

$$
\cdot H_{11}(\varphi, \psi)=\frac{1}{2} \frac{b_{11} g_{22}-2 b_{12} g_{12}+b_{22} g_{11}}{g_{11} g_{22}-g_{12}^{2}}
$$


где

$$
\begin{aligned}
& \mathrm{g}_{11}=\left(\frac{\partial \mathrm{x}}{\partial \varphi}\right)^{2}+\left(\frac{\partial \mathrm{y}}{\partial \varphi}\right)^{2}+\left(\frac{\partial \mathrm{z}}{\partial \varphi}\right)^{2}, \\
& \mathrm{~g}_{22}=\left(\frac{\partial \mathrm{x}}{\partial \psi}\right)^{2}+\left(\frac{\partial \mathrm{y}}{\partial \psi}\right)^{2}+\left(\frac{\partial \mathrm{z}}{\partial \psi}\right)^{2}, \\
& \mathrm{~g}_{12}=\frac{\partial \mathrm{x}}{\partial \varphi} \frac{\partial \mathrm{x}}{\partial \psi}+\frac{\partial \mathrm{y}}{\partial \varphi} \frac{\partial \mathrm{y}}{\partial \psi}+\frac{\partial \mathrm{z}}{\partial \varphi} \frac{\partial \mathrm{z}}{\partial \psi}
\end{aligned}
$$

$$
\begin{gathered}
\mathrm{b}_{11}=\frac{1}{\sqrt{\mathrm{g}_{11} \mathrm{~g}_{22}-\mathrm{g}_{12}^{2}}}\left(\frac{\partial^{2} \mathrm{x}}{\partial \varphi^{2}} \frac{\partial(\mathrm{y}, \mathrm{z})}{\partial(\varphi, \psi)}+\frac{\partial^{2} \mathrm{y}}{\partial \varphi^{2}} \frac{\partial(\mathrm{z}, \mathrm{x})}{\partial(\varphi, \psi)}+\frac{\partial^{2} \mathrm{z}}{\partial \varphi^{2}} \frac{\partial(\mathrm{x}, \mathrm{y})}{\partial(\varphi, \psi)}\right) \\
\mathrm{b}_{22}=\frac{1}{\sqrt{\mathrm{g}_{11} \mathrm{~g}_{22}-\mathrm{g}_{12}^{2}}}\left(\frac{\partial^{2} \mathrm{x}}{\partial \psi^{2}} \frac{\partial(\mathrm{y}, \mathrm{z})}{\partial(\varphi, \psi)}+\frac{\partial^{2} \mathrm{y}}{\partial \psi^{2}} \frac{\partial(\mathrm{z}, \mathrm{x})}{\partial(\varphi, \psi)}+\frac{\partial^{2} \mathrm{z}}{\partial \psi^{2}} \frac{\partial(\mathrm{x}, \mathrm{y})}{\partial(\varphi, \psi)}\right) \\
\mathrm{b}_{12}=\frac{1}{\sqrt{\mathrm{g}_{11} \mathrm{~g}_{22}-\mathrm{g}_{12}^{2}}}\left(\frac{\partial^{2} \mathrm{x}}{\partial \varphi \partial \psi} \frac{\partial(\mathrm{y}, \mathrm{z})}{\partial(\varphi, \psi)}+\frac{\partial^{2} \mathrm{y}}{\partial \varphi \partial \psi} \frac{\partial(\mathrm{z}, \mathrm{x})}{\partial(\varphi, \psi)}+\frac{\partial^{2} \mathrm{z}}{\partial \varphi \partial \psi} \frac{\partial(\mathrm{x}, \mathrm{y})}{\partial(\varphi, \psi)}\right)
\end{gathered}
$$

Используя вид частных производных (4) - (8), сразу получаем

$$
\begin{aligned}
& b_{11}=-\theta \sin ^{2} \psi-\theta_{\psi}^{\prime} \cos \psi \sin \psi-\theta_{\psi \psi}^{\prime \prime}, \\
& b_{22}=-\theta-\theta_{\psi \psi}^{\prime \prime}, \quad b_{12}=\theta_{\varphi}^{\prime} \operatorname{ctg} \psi-\theta_{\varphi \psi}^{\prime \prime}
\end{aligned}
$$

и

$$
\begin{gathered}
g_{11}=\frac{1}{\sin ^{2} \psi}\left(\theta \sin ^{2} \psi+\theta_{\psi}^{\prime} \cos \psi \sin \psi+\theta_{\varphi \varphi}^{\prime \prime}\right)^{2}+\left(\theta_{\varphi}^{\prime} \operatorname{ctg} \psi-\theta_{\varphi \psi}^{\prime \prime}\right)^{2}, \\
g_{22}=\left(\theta+\theta_{\psi \psi}^{\prime \prime}\right)^{2}+\frac{1}{\sin ^{2} \psi}\left(\theta_{\varphi}^{\prime} \operatorname{ctg} \psi-\theta_{\varphi \psi}^{\prime \prime}\right)^{2}, \\
g_{12}=-\left(\theta_{\varphi} \operatorname{ctg} \psi-\theta_{\varphi \psi}^{\prime \prime}\right)\left(2 \theta+\theta_{\psi \psi}^{\prime \prime}+\theta_{\psi}^{\prime} \operatorname{ctg} \psi+\theta_{\varphi \varphi}^{\prime \prime} \frac{1}{\sin ^{2} \psi}\right) .
\end{gathered}
$$


$g_{11} g_{22}-g_{12}^{2}=\frac{1}{\sin ^{2} \psi}\left(\left(\theta+\theta_{\psi \psi}^{\prime \prime}\right)\left(\theta \sin ^{2} \psi+\theta_{\psi}^{\prime} \cos \psi \sin \psi+\theta_{\psi \psi}^{*}\right)-\left(\theta_{\varphi}^{\prime} \operatorname{ctg} \psi-\theta_{\omega \psi}^{*}\right)^{2}\right)^{2}$

Подставляя полученные величины в (12) и (13), получим значения Гауссовой и средней кривизны соответственно.

Из предложений 1 и 2 вытекает следующее утверждение:

Теорема 1. Пусть функция $\theta(\varphi, \psi)$ на прямоугольнике $[0,2 \pi] \times[0, \pi]$ строго положительная и непрерывная вместе со своими производными до второго порядка включительно. Кроме того, функция $\mathrm{K}_{n}(\varphi, \psi)$ непрерывная строго положительная на $[0,2 \pi] \times[0, \pi]$ (при этом мы, естественно, считаем, что

$$
\left.\mathrm{K}_{\Pi}(\varphi, 0)=\mathrm{K}_{\Pi}(\varphi, 0+) \text { и } \mathrm{K}_{\Pi}(\varphi, \pi)=\mathrm{K}_{\mathbf{n}}(\varphi, \pi-0)\right) \text {. }
$$

Тогда уравнения (1) описывают замкнутую, строго выпуклую, ограниченную поверхность.

Замечание. Можно показать, что справедливо и обратное утверждение, то есть любая выпуклая, гладкая, замкнутая поверхность представима в виде (1), где функция $\theta(\varphi, \psi)$ на прямоугольнике $[0,2 \pi] \times[0, \pi]$ строго положительная и непрерывная вместе со своими производными до второго порядка включительно и при этом

$$
\left(\theta+\theta_{\psi \psi}^{*}\right)\left(\theta \sin ^{2} \psi+\theta_{\psi}^{\prime} \cos \psi \sin \psi+\theta_{\varphi \varphi}^{\prime \prime}\right)-\left(\theta_{\varphi}^{\prime} \operatorname{ctg} \psi-\theta_{\varphi \psi}^{\prime \prime}\right)^{2}>0 .
$$

В этом случае будем говорить, что функция $\theta(\varphi, \psi)$ удовлетворяет условиям $(*)$.

Теорема 1 и замечание $\mathbf{k}$ ней и обусловило наш интерес $\mathbf{k}$ поверхностям, представимым в виде (1). Доказательство обратного утверждения в существенной мере опирается на приведенное ниже утверждение.

В каждой точке поверхности П( $\varphi, \psi)$, определенной равенствами (1), сушествует единственная касательная плоскость. Через $\delta(\Pi, \varphi, \psi)$ обозначим расстояние от точки М до касательной плоскости к поверхности (1) в точке $\Pi(\varphi, \psi)$. $\Pi(\varphi, \psi)$.

Величину $\delta(\Pi, \varphi, \psi) \cdot$ называют опорной функцией поверхности

Предложение 3. Для любых $\varphi \in[0,2 \pi]$ и $\psi \in(0, \pi)$ опорная функция поверхности $\Pi(\varphi, \psi)$, определенной равенствами $(1)$, равна $\delta(\Pi, \varphi, \psi)=\theta(\varphi, \psi)$.

Доказательство. Подставляя в уравнение касательной плоскости

$$
n_{x}^{0}(x-x(\varphi, \psi))+n_{y}^{0}(y-y(\varphi, \psi))+n_{z}^{0}(z-z(\varphi, \psi))=0,
$$


координаты нормального вектора (3) и значения (1), получаем

$$
x \sin \psi \cos \varphi+y \sin \varphi \sin \psi+z \cos \psi-
$$

$-\sin \psi \cos \varphi\left(\theta \sin \psi \cos \varphi+\theta_{\psi}^{\prime} \cos \psi \cos \varphi-\theta_{\varphi}^{\prime} \frac{\sin \varphi}{\sin \psi}\right)-$

$-\sin \varphi \sin \psi\left(\theta \sin \psi \sin \varphi+\theta_{\psi}^{\prime} \cos \psi \sin \varphi+\theta_{\varphi}^{\prime} \frac{\cos \varphi}{\sin \psi}\right)-$

$$
-\cos \psi\left(\theta \cos \psi-\theta_{\psi}^{\prime} \sin \psi\right)=0
$$

или, что то же,

$$
x \sin \psi \cos \varphi+y \sin \varphi \sin \psi+z \cos \psi-\theta(\varphi, \psi)=0
$$

Расстояние от этой плоскости до начала координат равно $\theta(\varphi, \psi)$, что доказывает справедливость предложения 2.

При исследовании выпуклых поверхностей важную роль играк эквидистанты $\cdot \kappa$ ним. Дадим определение эквидистант, ограничившис спучаем замкнутых поверхностей.

$\varepsilon$-слоем поверхности $\Pi$ назовем объединение всех шаров радиуса $\varepsilon$ центрами, лежащими на поверхности $\Pi$, то есть $\mathrm{K}_{\varepsilon}(\Pi)=\bigcup_{M \in \Pi} \mathrm{B}_{\varepsilon}(\mathrm{M})$, гә $\mathrm{B}_{\varepsilon}(\mathrm{M})$ шар радиуса $\varepsilon \mathrm{c}$ центром в точке $\mathrm{M}$.

Внешнюю границу $\Pi_{\varepsilon}^{+} \varepsilon-$-ллоя $\mathrm{K}_{\varepsilon}(\Pi)$ будем называть внешней $\varepsilon$ эквидистантой поверхности $\Pi$, а внешнюю границу $\Pi_{\varepsilon}^{-}$(если она выпукле и лежит внутри области, ограниченной поверхностью П) назове

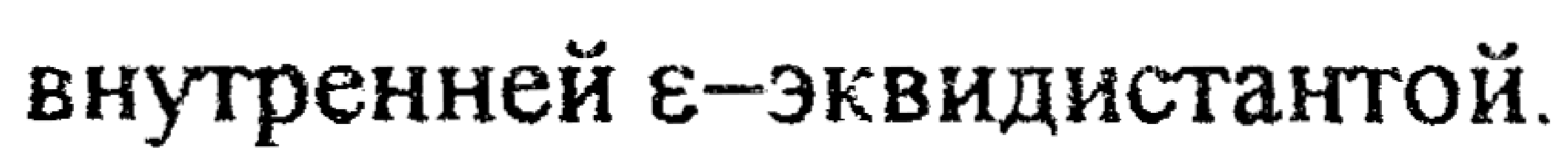

Теорема 2. Пусть для любых $\varphi \in[0,2 \pi]$ и $\psi \in(0, \pi)$ функция $\theta(\varphi, \iota)$

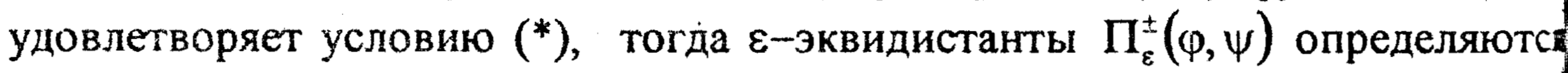
равенствами

$$
\left\{\begin{array}{c}
x_{\varepsilon}^{ \pm}=x_{\varepsilon}^{ \pm}(\varphi, \psi)=(\theta(\varphi, \psi) \pm \varepsilon) \sin \psi \cos \varphi+\theta_{\psi}^{\prime}(\varphi, \psi) \cos \psi \cos \varphi-\theta_{\varphi}^{\prime}(\varphi, \psi) \frac{\sin \varphi}{\sin \psi} \\
y_{\varepsilon}^{ \pm}=y_{\varepsilon}^{ \pm}(\varphi, \psi)=(\theta(\varphi, \psi) \pm \varepsilon) \sin \psi \sin \varphi+\theta_{\psi}^{\prime}(\varphi, \psi) \cos \psi \sin \varphi+\theta_{\varphi}(\varphi, \psi) \frac{\cos \varphi}{\sin \psi} \\
z_{\varepsilon}^{ \pm}=z_{\varepsilon}^{ \pm}(\varphi, \psi)=(\theta(\varphi, \psi) \pm \varepsilon) \cos \psi-\theta_{\psi}^{\prime}(\varphi, \psi) \sin \psi
\end{array}\right.
$$


при люб́ом $\varepsilon$ для внешней эквидистанты и при $\varepsilon$ таком, что внутренняя граница $\mathrm{K}_{\varepsilon}(\mathrm{I})$ лежит в области, ограниченной $\Pi$, и является вытуклой для внутренней эквидистанты.

Доказательство.. Действительно, в силу условий, наложенных на функцию $\theta(\varphi, \psi)$; все первые частные производные (4)-(8) непрерывны и, следовательно, $\varepsilon$-эквидистанты данной поверхности можно определить следующим образом:

$$
\left\{\begin{array}{c}
x_{\varepsilon}^{ \pm}(\varphi, \psi)=x(\varphi, \psi) \pm \varepsilon \frac{\frac{\partial(y, z)}{\partial(\varphi, \psi)}}{\sqrt{g_{11} g_{22}-g_{12}^{2}}} \\
y_{\varepsilon}^{ \pm}(\varphi, \psi)=y(\varphi, \psi) \pm \varepsilon \frac{\frac{\partial(z, x)}{\partial(\varphi, \psi)}}{\sqrt{g_{11} g_{22}-g_{12}^{2}}} \\
z_{\varepsilon}^{ \pm}(\varphi, \psi)=z(\varphi, \psi) \pm \varepsilon \frac{\partial(x, y)}{\sqrt{g_{11} g_{22}-g_{12}^{2}}}
\end{array}\right.
$$

Используя значения функциональных определителей, отсюда и из (1) после естественных, но громоздких вычислений, получаем требуемое утверждение.

В заключение приведем еще одно утверждение.

Предложение 5. Пусть для любых $\varphi \in[0,2 \pi]$ и $\psi \in(0, \pi)$ функция $\theta(\varphi, \psi)$ удовлетворяет условию $\left({ }^{*}\right)$, тогда площадь поверхности П определяется равенством

$$
S(\Pi)=\int_{0}^{2 \pi} d \varphi \int_{0}^{\pi} \frac{\sin \psi}{K_{\Pi}(\varphi, \psi)} d \psi=\int_{0}^{2 \pi} d \varphi \int_{0}^{\pi}\left(\rho+\rho_{\psi \psi}^{\prime \prime}\right)\left(\rho \sin \psi+\rho_{\psi}^{\prime} \cos \psi\right) d \psi .
$$

Доказательство этого факта легко получается из вида дифференџиала площади

$$
\mathrm{dS}(\varphi, \psi)=\sqrt{\mathrm{g}_{11} \mathrm{~g}_{22}-\mathrm{g}_{12}^{2}} \mathrm{~d} \varphi d \psi
$$

для поверхности,определенной равенствами (1).

1. Адамар Ж. Дифференциалькая геометрия . М.,1938.

2. Лигун А.А., Шумейко А.А. О геометрии выпуктых кривых // Тез. междунар. конф.

"Теория приблжжений и гармоническнй анализ". Тула, 1988. С. 156. 